\title{
DIFERENCIAS EN LA EXPERIENCIA SUBJETIVA ENTRE DEPRESION \\ UNIPOLAR Y BIPOLAR
}

\begin{abstract}
Autores:
Andrés Bustos. MD. Residente de Psiquiatría Universidad del Rosario.

Marco Fierro. MD. Psiquiatra. M Phil. Profesor de psiquiatría. Universidad del Rosario.

Carlos Molina. MD. Psiquiatra Epidemiólogo. Docente Investigador Universidad Externado de Colombia. Catedrático Universidad del Rosario y Unisanitas. Coordinador Subcomité de S.M.P y S ACP.
\end{abstract}

Dirección: Calle 103 A \# 21-49. Bogotá - Colombia

Titulillo: Sin titulillo. Para identificación de las páginas impares puede utilizarse el mismo título.

Para escribir este artículo no recibimos ninguna financiación.

Sin conflictos de interés. 


\section{DIFERENCIAS EN LA EXPERIENCIA SUBJETIVA ENTRE DEPRESION}

\section{UNIPOLAR Y BIPOLAR}

\section{RESUMEN.}

Introducción. Cada vez es más importante poder distinguir la depresión unipolar de la bipolar, pues hay diferencias en el tratamiento y el pronóstico.

Objetivo. Buscar diferencias en la experiencia subjetiva (de primera persona) entre depresión unipolar y bipolar.

Método. Se utilizó una entrevista semiestructurada de orientación fenomenológica basada en el Examen de la experiencia anómala del self (EASE).

Resultados. En la depresión unipolar el estado de ánimo predominante es la tristeza y la experiencia corporal es de un cuerpo que se siente más cómodo con la quietud que con la actividad, parecido a la pereza de la vida cotidiana. En la depresión bipolar el estado de ánimo predominante es de apagamiento emocional. La experiencia corporal es de un cuerpo pesado, cansado, un elemento que se interpone entre los deseos de actuar y la realización de las acciones y que se vuelve un obstáculo para el movimiento. Además, en la depresión bipolar hay mayor bradipsíquia, dificultad para concentrarse y desesperanza que en la unipolar.

Conclusión. Se encontraron diferencias cualitativas en el estado de ánimo predominante y la experiencia corporal entre la depresión unipolar y la bipolar.

Palabras clave: trastorno bipolar, depresión unipolar, depresión bipolar, fenomenología, síntomas. EASE. 


\title{
Title: Differences in subjective experience between unipolar and bipolar depression
}

\begin{abstract}
Introduction. It is increasingly important to make distinction between bipolar and unipolar depression because treatment and prognosis are different.

Objective. Find differences in subjective experience (first person) between unipolar and bipolar depression. .

Method. We used a phenomenological-oriented semi-structured interview based on Examination of Anomalous Self-Experience (EASE).

Results. The predominant mood in unipolar depression is sadness and bodily experience is of a body that feels more comfortable with the stillness than activity, like laziness of everyday life. The predominant mood in bipolar depression is emotional quenching. The bodily experience is of a heavy, tired body; an element that inserts between the desires of acting and performing actions and becomes an obstacle to the movement. Furthermore, bradypsychia, concentration difficulty and hopelessness were greater in bipolar than unipolar depression.
\end{abstract}

Conclusion. Qualitative differences in the predominant mood and bodily experience were found between bipolar and unipolar depression.

Keywords: Bipolar Disorder, Bipolar Depression, Unipolar Depression, Phenomenology, Symptoms, EASE. 


\section{INTRODUCCION.}

El episodio depresivo es el elemento fundamental para hacer el diagnóstico de trastorno depresivo mayor, pero puede también presentarse en el trastorno bipolar. Cuando hace parte del trastorno depresivo mayor se le llama también depresión unipolar y cuando ocurre en el marco del trastorno bipolar se le denomina depresión bipolar ${ }^{(1-3)}$.

En el DSM IV TR no hay criterios que permitan distinguir el episodio depresivo unipolar del bipolar. Sin embargo, cada vez se ha vuelto más importante establecer esa diferencia, pues al hacerla en forma temprana y certera, se puede evitar un mayor número de recurrencias, tratamientos farmacológicos inadecuados ${ }^{(4-11)}$, aparición de comorbilidades (12-16) y complicaciones propias de la enfermedad.

Los episodios depresivos bipolares se han relacionado con una mayor incidencia de suicidio $(10-15 \%)$ que los unipolares ${ }^{(17-22)}$. También con trastornos de ansiedad y un mayor consumo de alcohol y sustancias psicoactivas ${ }^{(19)}$.

Clínicamente, los episodios depresivos de trastorno bipolar aparecen a una edad más

temprana ${ }^{(3,23-25)}$. Han sido descritos como episodios recidivantes, de duración breve, con síntomas atípicos, hipersomnio, ansiedad, quejas somáticas, irritabilidad e incluso psicosis $(2,3,13,22,26-30)$.

En cuanto a la historia familiar se ha encontrado clara correspondencia entre depresión unipolar y bipolar con antecedentes de trastorno depresivo mayor y trastorno bipolar 
respectivamente. ${ }^{(23,31,32)}$. Sin embargo, debido a la existencia de depresiones bipolares subdiagnosticadas o diagnosticadas como unipolares, se recomienda tomar los antecedentes de psicosis y/o suicidio como indicador de depresión bipolar aún si el diagnostico de trastorno bipolar no se hubiese hecho ${ }^{(33)}$.

La exploración de las experiencias subjetivas (en primera persona) rara vez se ha utilizado para establecer diferencias entre la depresión unipolar y la bipolar, si bien ha dado buenos resultados en el estudio de la esquizofrenia, especialmente del pródromo ${ }^{(34-38)}$.

\section{MATERIALES Y METODOS.}

Se realizó una investigación de tipo cualitativo, exploratorio, de las experiencias subjetivas (de primera persona) de un grupo de pacientes con depresión unipolar y bipolar. Se utilizó entrevistas en profundidad de orientación fenomenológica, basadas en un guión, aplicadas a todos los participantes, seguidas de la interpretación comparativa de los hallazgos en términos fenomenológicos ${ }^{(39)}$.

Se incluyeron en el estudio 12 pacientes, 3 hombres y 9 mujeres mayores de 18 años que cumplieron criterios para un episodio depresivo moderado a severo sin síntomas psicóticos, (clasificación DSM IV TR). Todos ellos también reunieron criterios diagnósticos de trastorno bipolar (7) o trastorno depresivo mayor (5). Antes de la realización de la entrevista cada paciente fue informado sobre el objetivo de la misma y aceptó participar voluntariamente en la investigación. 
La entrevista fue realizada en un ambiente empático en el que se animó al (la) paciente a hablar de su experiencia sin ninguna restricción. Para tal fin, a cada participante, se le explicó que la información relevante era el conjunto de experiencias vividas durante el episodio depresivo y se le pidió que las describa con la mayor claridad posible. Por parte del entrevistador, se evitaron las reacciones verbales y preverbales que pudieran dar lugar a que el entrevistado se sienta juzgado, atemorizado o desconfiado. También se le informó al paciente que en caso de no comprender claramente una pregunta pida aclaraciones hasta que logre captar el sentido de lo que se indaga. Las preguntas fueron escritas en un guión para de esta forma seguir siempre una secuencia y no olvidar la evaluación de ninguno de los ítems. Se dejó la posibilidad de hacer variaciones en el orden, dependiendo de que las respuestas previas hayan cubierto algún aspecto de la exploración ${ }^{(40)}$.

La entrevista utilizada en este estudio se basó en la desarrollada por J. Parnas y cols. en la universidad de Copenhagen, Dinamarca, denominada Examen de la experiencia anómala del self (EASE). Se trata de una herramienta de exploración fenomenológica que funciona como una lista de chequeo de síntomas y se aplica como una entrevista semiestructurada. Consiste en cinco dominios, cada uno de ellos compuesto por un número diferente de preguntas, las cuales buscan establecer la presencia de las experiencias anómalas y la intensidad de las mismas. Cada dominio y subdominio esta explicado en un documento anexo, de tal manera que el clínico pueda reconocer las características propias de cada experiencia y ubicarla en alguno de los subdominios después de escuchar las respuestas del paciente. 
Se tomo la EASE como modelo, obviando los dominios y subdominios específicos orientados a indagar por la psicosis, y se adaptó para aplicarla a pacientes con un episodio depresivo sin síntomas psicóticos. Teniendo en cuenta que la depresión se manifiesta en experiencias no sólo mentales sino también corporales y en la manera de ser y estar en el mundo, se seleccionó cuatro dominios con sus respectivos subdominios. Los dominios son: 1. la experiencia emocional, 2. la experiencia con el propio cuerpo, 3. la cognición y el flujo de conciencia y 4. la proyección en el tiempo o perspectiva de futuro. Cada dominio y subdominio está descrito y ejemplificado en un documento que organiza la entrevista, el cual debe ser bien conocido por el entrevistador antes de aplicarlo. Cada entrevista se registró en un formato audiovisual; para ello el paciente estuvo de acuerdo y firmó un consentimiento informado. Después de una primera aproximación a campo se efectuaron los ajustes necesarios a la entrevista con el fin de acercarla a la experiencia en primera persona y hacerla más entendible a los indagados. Se realizó una verificación de los relatos y de la construcción de sentido en el curso de las entrevistas para de esta manera objetivar las apreciaciones sobre la experiencia del paciente que hizo el entrevistador.

\section{RESULTADOS.}

A partir de las entrevistas realizadas, se pudo apreciar:

\section{EXPERIENCIA AFECTIVA PREDOMINANTE.}

Con respecto a la experiencia afectiva predominante hay diferencias de importancia. En la depresión bipolar la tristeza no es el estado de ánimo predominante; en su lugar se instala uno que quizá pueda ser descrito mejor con el término apagamiento emocional. Los pacientes se refieren a él con expresiones como dificultad para sentír y vacío de 
sentimientos. El apagamiento se instala en el primer plano de la conciencia y afecta de forma significativa toda la vida mental.

- PL. "Todo el tiempo apagada emocionalmente. Es que no hay nada que lo emocione a uno [...] Era imposible para mi decir si estoy emocionada o estoy triste o estoy feliz, es que no había emoción, es plano. No es tristeza, es un sentimiento plano. Por ejemplo: se muere mi tío, que embarrada pero bueno; se enferma mi perro, que embarrada, pero bueno. Vámonos de rumba a pasarla cheverisimo; no, ¿para qué?; que mamera, me da igual [...] Antes de apagarme estoy más irritable y ya sé que me viene la crisis".

- IN. "Apagada emocionalmente, como sin vida todo el tiempo".

- YE. "Es un vacío de sentimientos que duele. Es distinto a lo que pasa cuando a uno las cosas no le importan. En este caso (estando con depresión bipolar), la emoción no llega con nada, ni siquiera la tristeza. Es como estar muerto.

- LU. "Es como si no pudiera sentir, como si no sintiese nada, como si hubiese perdido la capacidad de sentir, todo da igual".

Conviene aclarar que la experiencia afectiva de la depresión bipolar no es una ausencia de sentimientos. Hay un sentimiento claro y doloroso de apagamiento. El paciente se percata, se da cuenta del mismo y lo sufre. El mundo que aparece ante él no es plano sino con menos vitalidad, es menos atractivo e interesante. 
En la depresión unipolar, el estado de ánimo se asemeja a la tristeza cotidiana y a la que se observa en un duelo. Ese estado de ánimo es menos invasivo que el apagamiento emocional, pues le permite al afectado interesarse un poco más en realizar ciertas actividades y tener momentos en que la tristeza pasa a un segundo plano. De hecho, la mayoría de los pacientes entrevistados negó experienciar un sentimiento diferente o más notorio que la tristeza.

- TL. "Me siento mal. Me falta todo, estoy cansada, Me he sentido triste, mucho, lloro todos los días". Con respecto al apagamiento emocional dice: "no sentir nada dura muy poquito tiempo, es pasajerito".

- ES."Es una tristeza muy fuerte. Soy capaz de trabajar y seguir con las cosas del día a día, pero la tristeza está siempre detrás”

De otra parte, en la depresión bipolar, cuando la persona se percata del apagamiento emocional suele experimentar ansiedad y rabia, junto con pensamientos reiterados de autorreproche y culpa por no poder retornar a su estado habitual.

- PL: "me doy cuenta de mi estado y siento rabia por lo que me sucede y no poder cambiarlo"

Si bien el paciente es quien experiencia el tinte cualitativo de su estado de ánimo, no es fácil para él describirlo, expresarlo en palabras y hacerlo entendible a los demás. En cambio, cuando se le pregunta explícitamente acerca de sentimientos de tristeza y 
apagamiento emocional, la mayoría son capaces de hacer esa distinción con relativa facilidad. Una paciente pudo hablar claramente de esa diferencia, pues al final del episodio de depresión bipolar, cuando ya había mejorado de la misma, afrontó una situación vital que le generó gran tristeza. Comprendía que la tristeza era ocasionada por circunstancias que en algún momento finalizarían o bien ella se adaptaría a las mismas. No sucedía así con la experiencia de la depresión bipolar, cuyo comienzo y fin, en su momento, no veía claro y temía que se prolongara por tiempo indefinido.

- PL cuando estaba triste. "Estoy triste porque me voy a ir en una semana y tengo que dejar a mi hijo por tres meses. Me voy a ir, voy a dejarlo, es más como esa ansiedad de separación que me pone triste, que me pone a llorar, pero no es algo que uno diga no lo voy a superar, sino que en el momento uno no puede pensar bien. Es más la circunstancia”.

- PL cuando tenía depresión bipolar. "Uno ve como la gente se ríe, canta, baila, y uno está ahí, como inerte. Nada resuena, nada resalta. Hay indiferencia ante lo que pasa en el mundo".

El llanto figura con más frecuencia en la depresión unipolar que la bipolar. En la última está relacionado con el desespero ante una situación que parece no tener fin.

- PL. "No es una llorada de desahogo, sino de desespero". 
Con frecuencia, los familiares y amigos de los pacientes observan cambios en la expresión facial, sobre todo en la depresión bipolar.

- CC. "La familia nota cuando estoy enferma, me dicen que cambia mi cara, que me veo decaída y casi no se me obserban muestras de emociones".

- JF."La gente que me conoce me dice que cambia mi cara, la rapidez con que me muevo y hasta la voz. Cuando estoy deprimido hablo como si no tuviera fuerza en la voz, no subo ni bajo, como una voz casi plana".

- PL "Mi hermana reconoce que estoy apagada emocionalmente cuando me nota monosilábica y con tendencia al mutismo. Otros, como el jefe o los compañeros de trabajo dicen que me ven rara. Antes de apagarme, las cosas me molestan más, como la voz de la gente".

\section{EXPERIENCIA DEL CUERPO}

En la vida cotidiana, el cuerpo es fenomenológicamente silencioso. Por ejemplo, al caminar uno no se da cuenta de los movimientos de las piernas; es más, se puede ir concentrado en los pensamientos sin percatarse de que se ha recorrido una gran distancia. Al trabajar en un computador, los movimientos de los dedos al oprimir las teclas se trasladan al transfondo, pasando la pantalla a ocupar el primer plano de la conciencia. En los dos casos, el movimiento de las piernas y los dedos se vuelve transparente; está allí, pero pasa desapercibido. 
Cuando hay dolor, la situación cambia radicalmente. Los movimientos del cuello, al girar, habitualmente pasan desapercibidos, pero si se sufre tortícolis, el dolor y la rigidez de los músculos hacen que el movimiento sea experienciado con claridad: se siente la resistencia y se hace necesario hacer un esfuerzo cuidadoso para mitigar las molestias. Se pone atención a unos movimientos que en otras circunstancias no la requieren.

El cuerpo es percibido de forma diferente en la depresión bipolar y la unipolar. En la bipolar los pacientes describen su cuerpo como un obstáculo para el movimiento y la realización de la acciones, dificultándose incluso las actividades cotidianas y el desplazamiento. El cuerpo se siente pesado, como un lastre, un elemento que se interpone entre los deseos de actuar y la realización de las acciones. Es un cuerpo que está cansado todo el tiempo, prácticamente enfermo. Es también un cuerpo más torpe, con menos soltura y ductilidad. Se parece a la experiencia de cansancio después de realizar ejercicio intenso y prolongado, pero sin el bienestar generado por el ejercicio. Esta experiencia del cuerpo da lugar a que la persona pueda permanecer todo el día en cama sin que aparentemente esté enferma ni tenga un dolor específico.

- CQ. "Siento el cuerpo pesado, con movimientos lentos. En todo me demoro más y es con más dificultad. Hasta soy más torpe con lo que hago, como que no coordino bien, Me cuesta mucho terminar lo que empiezo".

- IN. "Me duele por todo lado; un peso aquí (señala el cuello). Se me dificulta mucho hacer actividades cotidianas, subir y bajar de los buses". 
- CC. "Es difícil hasta caminar. Veo las distancias muy lejos. Antes caminaba varias cuadras sin problema, ni lo sentía. Ahora, me cuesta mucho, me canso. El cuerpo no responde igual".

En la depresión unipolar, el cuerpo no se experiencia de forma tan notoria como en la bipolar. Más bien, el cuerpo se sintoniza con la tristeza, casi se podría decir que es un cuerpo triste. La experiencia del cuerpo es parecida a la que se tiene en la pereza. Es un cuerpo que se siente más cómodo con la quietud que con la actividad. No representa un obstáculo tan grande para el movimiento como en el caso de la depresión bipolar, pero se cansa con facilidad una vez entra en acción. El resultado en ambos tipos de depresión es el empleo de mayor tiempo en realizar las actividades.

- QM. "Voy a trabajar y hago muy poco. Comienzo una cosa y me aburre, me canso y la dejo. Sé que tengo que hacer muchas cosas, pero es difícil comenzar alguna. Veo varias páginas de internet, leo un poco de cada una, veo si tengo llamadas, así se pasa el tiempo, y lo que es del trabajo casi nada".

- OG. "Me levanto, no me animo a bañarme; digo, más tarde. A veces no me cepillo los dientes. Uno está dejado, como dice la gente. Es un desánimo, como pereza, pero no es exactamente pereza. Es como si fuera mejor quedarse viendo televisión sin hacer nada, sin hacer ningún esfuerzo". 


\section{COGNICIÓN Y FLUJO DE LA CONCIENCIA}

En la depresión bipolar el pensamiento se enlentece más que en la unipolar y la concentración se afecta en mayor medida. Las personas sienten dificultad para organizar las ideas y seguir el curso del pensamiento dirigido a un propósito (por ejemplo, resolver un problema), tanto que se vuelve notorio para los observadores. Cuando comienzan a realizar una tarea como leer, les es difícil concentrarse y culminarla. El pensamiento se centra en el estado que padecen y los síntomas que presentan. Además, experiencian mayor culpa y desesperanza que en la unipolar. Suelen decir "no he logrado nada por mi esfuerzo, por mis meritos y las cosas buenas que tengo no las merezco”. Al compararse con los demás encuentran que están menos vivos, se sienten incapaces de realizar las actividades cotidianas como lo hacían habitualmente.

- PL. "Los pensamientos no fluyen. Si este que está aquí me está mirando es porque yo no he podido terminar lo que tengo que hacer. Siempre es denigrándome a mí misma, es como un auto sabotaje [...] Siento culpa por no poder organizar el trabajo ni rendir en él. Todo es una cadena, no puedo pensar positivo [...] Me imagino haciendo las cosas mal. Termino no haciendo las cosas por eso. Todo el tiempo pienso que me va ir mal, que está mal”.

- CQ. "Siento como un bloqueo, no tengo las mismas ideas que tenía antes. Me cuesta dejar de pensar en cómo me siento y concentrarme en otras cosas o tener pensamientos de otras cosas que no sean mi depresión. Decidí tomar otro trabajo para que me sustente lo que estoy haciendo ahora, pero me preocupa lo que la gente piense de mi; por dejar, entre comillas, tirado un proyecto que ya estaba ejecutando, cuando no tiene porque importarme lo que digan, si son mis decisiones". 


\section{PERSPECTIVAS DEL FUTURO.}

La desesperanza es más intensa en la depresión bipolar que en la unipolar. Las personas sienten que no son capaces de continuar realizando las mismas actividades, pues han perdido sus capacidades de una forma casi irreversible. Se sienten con mayor edad que la cronológica y vislumbran sus caminos cerrados y sin opciones.

- CC. "Hay un momento que uno piensa que hasta ahí no más fue. Uno piensa que ya lo que uno viva es porque mi dios lo deja y tiene que aceptarlo".

- JF. Un hombre de 47 años sin problemas laborales, económicos ni familiares, habitualmente tranquilo y optimista, en medio de la depresión bipolar, anotaba: "creo que no voy a poder seguir en el trabajo, hay mucha gente joven que sí tiene energía y puede hacerlo bien, en cambio yo ya no puedo. Veo que no podré darles educación a mis hijos ni tendré más oportunidades de trabajar”

\section{CONCLUSIONES.}

Mediante entrevistas en profundidad guiadas fenomenológicamente se encontró algunas diferencias importantes entre la depresión bipolar y la unipolar. En la depresión bipolar el estado de ánimo predominante es el apagamiento emocional, y el cuerpo se experiencia pesado, francamente cansado, como un obstáculo para el movimiento. En la depresión unipolar el estado de ánimo predominante es la tristeza, y la experiencia del cuerpo se parece a la pereza cotidiana. De otra parte, en la depresión bipolar hay mayor bradipsíquia, dificultad para concentrarse y desesperanza que en la unipolar. 
Estos hallazgos de tipo fenomenológico se suman a toda una serie de datos epidemiológicos y clínicos, y sirven al propósito de establecer diferencias entre los dos tipos de depresión desde el momento mismo que el paciente acude a la consulta. 


\section{REFERENCIAS.}

(1) Cuellar A, Johnson S, Winters R. Distinctions between bipolar and unipolar depression. Clinical Psychology Review. 2005;25:307-339.

(2) Ohmae S. The difference between depression and melancholia: two distinct conditions that were combined into a single category in DSM-III. Seishin Shinkeigaku Zasshi. (Psychiatria et neurologia). 2012;114: 886-905.

(3) Akiskal HS, Bourgeois ML, Angst J, Post R, Möller H, Hirschfeld R. Re-evaluating the prevalence of and diagnostic composition within the broad clinical spectrum of bipolar disorders. J Affect Disord. 2000;59(Suppl 1):5-30.

(4) Henry C. Severe Depression: specific features of bipolar disorder. Encephale. 2009; 35 (S7):261-3

(5) Li CT, Bai YM, Huang YL, Chen YS, Chen TJ, Cheng JY, Su TP. Association between antidepressant resistance in unipolar depression and subsequent bipolar disorder: cohort study. Br J psychiatry. 2012;200:45-51.

(6) Nivoli A, Colom F, Murru A, Pacchiarotti I, Castro-Loli P, González-Pinto A, Fountoulakis K, Vieta E. New treatment guidelines for acute bipolar depression: A systematic review. J Affective Disorders. 2011;129:14-26

(7) Fountoulakis KN, Vieta E. Treatment of bipolar disorder: a systematic review of available data and clinical perspectives. The International Journal of Neuropsychopharmacology. 2008;11:999-1029

(8) Saatcioglu O, Erim R, Tomruk N, Oral T, Alpay N. Antidepressant-associated mania or hypomania: A comparison with personality and bipolarity features of bipolar I disorder. J Affective Disorders. 2011;134:85-90 
(9) Scott E, Hermens D, Naismith S, Guastella A, Regt T, White D, Lagopoulos J, Hickie I. Distinguishing young people with emerging bipolar disorders from those with unipolar depression. Journal of Affective Disorders (2012), http://dx.doi.org/10.1016/j.jad.2012.06.031

(10) Soares-Weiser K, Bravo V Y, Beynon S, Dunn G, Barbieri M, Duffy S, Geddes J, Gilbody S, Palmer S, Woolacott N. A systematic review and economic model of the clinical effectiveness and cost-effectiveness of interventions for preventing relapse in people with bipolar disorder. Health Technol Assess. 2007;11(39):1-226.

(11) Correa R, Akiskal H, Gilmer W, Nierenberg AA, Trivedi M, Zisook S. Is unrecognized bipolar disorder a frequent contributor to apparent treatment resistant depression?. J Affect Disord. 2010;127(1-3):10-8.

(12) Moreno C, Hasin DS, Arango C, Oquendo MA, Vieta E, Liu S, Grant BF, Blanco C. Depression in bipolar disorder versus major depressive disorder: results from the National Epidemiologic Survey on Alcohol and Related Conditions. Bipolar Disord. 2012;14:271-82 (13) Gan Z, Diao F, Wei Q, Wu X, Cheng M, Guan N, Zhang M, Zhang J. A predictive model for diagnosing bipolar disorder based on the clinical characteristics of major depressive episodes in Chinese population. J Affect Disord. 2011;134:119-25.

(14) Xu G, Lin K, Rao D, Dang Y, Ouyang H, Guo Y, Ma J, Chen J. Neuropsychological performance in bipolar I, bipolar II and unipolar depression patients: A longitudinal, naturalistic study. J Affect Disord. 2012;136: 328-339

(15) Angst J, Azorin JM, Bowden CL, Perugi G, Vieta E, Gamma A, Young AH; BRIDGE Study Group. Prevalence and characteristics of undiagnosed bipolar disorders in patients with a major depressive episode: the BRIDGE study. Arch Gen Psychiatry. 2011;68:791-8. 
(16) van der Werf-Eldering MJ, Schouws S, Arts B, Jabben N. Cognitive dysfunction in bipolar disorder: determinants and functional outcome. Tijdschr Psychiatr. 2012;54:70918.

(17) McIntyre RS, Muzina DJ, Kemp DE, Blank D, Woldeyohannes HO, Lofchy J, et al. Bipolar disorder and suicide: research synthesis and clinical translation. Curr Psychiatry Rep. 2008;10:66-72.

(18) Bottlender R, Jäger M, Strauss A, Möller HJ. Suicidality in bipolar compared to unipolar depressed in patients. Eur Arch Psychiatry Clin Neurosci. 2000;5:257-61.

(19) Goldberg JF, Garno JL, Portera L, León AC, Kocsis JH, Whiteside JE. Correlates of suicidal ideation in dysphoric mania. J Affect Disord. 1999;56:75-81

(20) Gonda X, Pompili M, Serafini G, Montebovi F, Campi S, Dome P, Duleba T, Girardi P, Rihmer Z. Suicidal behavior in bipolar disorder: Epidemiology, characteristics and major risk factors Journal of Affective Disorders (2012), http://dx.doi.org/10.1016/j.jad.2012.04. (21) Tsai SY, Kuo Cj, Chen CC, Lee HC. Risk factors for completed suicide in bipolar disorder. J Clin Psychiatry. 2002 63:469-476

(22) Akiskal HS, Benazzi F, Perugi G, Rihmer Z. Agitated "unipolar" depression reconceptualized as a depressive mixed state: implications for the antidepressant-suicide controversy. J Affect Disord. 2005;85:245-58

(23) Bowden C. A different depresion: clinical distinctions between bipolar and unipolar depression. J Affect Disord 2005;84:117-25.

(24) Mitchell PB, Goodwin GM, Johnson GF, Hirschfeld RM. Diagnostic guidelines for bipolar depression: a probabilistic approach. Bipolar Disord. 2008;10:144-52.

(25) Sanchez P R, Rodríguez L J, Jaramillo L. Edad de inicio como marcador de subtipos de enfermedad maniacodepresiva. Rev. Colomb. Psiquiat. 2012; 41:576-87. 
(26) El-Mallakh R, Ghaemi N. Depresión Bipolar: Guía completa Waashington DC: American Psychiatric Publishing; 2006.

(27) Maj M, Pirozzi R, Magliano L, Bartol L. Agitated Depression in Bipolar I Disorder: Prevalence, Phenomenology, and Outcome. Am J Psychiatry 2003;160:2134-2140

(28) Roy H. Perlis, M.D. Eileen Brown, Ph.D. Robert W. Baker, M.D. Andrew A. Nierenberg, M.D. Clinical Features of Bipolar Depression Versus Major Depressive Disorder in Large Multicenter Trials Am J Psychiatry 2006;163:225-231

(29) Lövdahl H, Andersson S, Hynnekleiv T, Malt U. The phenomenology of recurrent brief depression with and without hypomanic features. J Affect Disord. 2009;112:151-164

(30) Judd L, Schettler P, Akiskal H, Coryell W, Fawcett J, Fiedorowicz J, Solomon D, Keller M. Prevalence and clinical significance of subsyndromal manic symptoms, including irritability and psychomotor agitation, during bipolar major depressive episodes. J Affect Disord. 2012;138:440-448

(31) Ghaemi S, Saggese J, Goodwin F. Diagnosis of Bipolar Depression. En: Mallakh R, Ghaemi S (Eds). Bipolar Depression: A comprehensive Guide. Washington DC: American Psychiatric Publishing; 2006.p.3-36.

(32) Craddock N, Forty L. Genetics of affective (mood) disorders. Eur J Hum Genet 2006;14: 660-8.

(33) Leyton F, Barrera Á. El diagnóstico diferencial entre la Depresión Bipolar y la Depresión Monopolar en la práctica clínica. Rev Med Chile. 2010;138:773-779.

(34) Parnas J, Møller P, Kircher T, Thalbitzer J, Jansson L, Handest P, Zahavi D. EASE: Examination of Anomalous Self-Experience. Psychopathology 2005;38:236-258.

(35) Merleau-Ponty M. Phenomenology of Perception. London: Routledge and Kegan; 1962. 
(36) García E. Fenomenología del cuerpo vivido y filosofía del viviente: M. Merleau-Ponty y G. Canguilhem. Acta fenomenológica latinoamericana. 2009;3:523-538.

(37) Fuchs T. Corporealized and disembodied minds: A Phenomenological View of the body in Melancholia and Schizophrenia. Philosophy, Psychiatry and Psychology. $2005 ; 12,2: 95-107$.

(38) Gallagher S, Zahavi D. The Phenomenological Mind: An Introduction to Philosophy of Mind and Cognitive Science. London and New York: Routledge. Taylor \& Francis Group e-Library; 2008

(39) Botero J. Fenomenología. En Hoyos LE (Ed). Lecciones de filosofía, Bogotá: Universidad Externado de Colombia/Universidad Nacional; 2003.p.311-332.

(40) Valles M. Técnicas cualitativas de investigación social 3ra reimpresión. Madrid: Síntesis; 2003. 